\title{
Non-abelian infrared divergences on the celestial sphere
}

\author{
Lorenzo Magnea ${ }^{a, b}$ \\ ${ }^{a}$ Theoretical Physics Department, CERN, \\ CH-1211 Geneva 23, Switzerland \\ ${ }^{b}$ Dipartimento di Fisica and Arnold-Regge Center, Università di Torino, \\ and INFN, Sezione di Torino, \\ Via P. Giuria 1, I-10125 Torino, Italy \\ E-mail: lorenzo.magnea@unito.it
}

ABSTRACT: We consider the infrared factorisation of non-abelian multi-particle scattering amplitudes, and we study the form of the universal colour operator responsible for infrared divergences, when expressed in terms of coordinates on the 'celestial sphere' intersecting the future light-cone at asymptotic distances. We find that colour-dipole contributions to the infrared operator, to all orders in perturbation theory, have a remarkably simple expression in these coordinates, with scale and coupling dependence factorised from kinematics and colour. Generalising earlier suggestions in the abelian theory, we then show that the infrared operator can be computed as a correlator of vertex operators in a conformal field theory of Lie-algebra-valued free bosons on the celestial sphere. We verify by means of the OPE that the theory correctly predicts the all-order structure of collinear limits, and the tree-level factorisation of soft real radiation.

Keywords: Scattering Amplitudes, Perturbative QCD, Conformal Field Theory

ARXIV EPRINT: 2104.10254 


\section{Contents}

1 Introduction 1

2 Non-abelian soft anomalous dimensions: a primer 4

$\begin{array}{lll}3 & \text { Colour correlations on the celestial sphere } & 7\end{array}$

4 A theory of Lie-algebra-valued free bosons $\quad 8$

$\begin{array}{llr}5 & \text { Some open questions } & 16\end{array}$

$\begin{array}{llr}6 & \text { Perspectives } & 18\end{array}$

\section{Introduction}

The problem of infrared divergences in gauge theory amplitudes and cross sections has been studied for close to a century, starting with the seminal work of Bloch and Nordsieck in QED [1], and solutions involving an increasing degree of generality and depth have been put forward across the decades. One class of proposed solutions is based upon the idea of carefully constructing well-defined (and thus finite) observable quantities, in the spirit of the KLN theorem [2,3]. Within this framework, one accepts that $S$-matrix elements are illdefined in massless theories, introduces an infrared regulator, and verifies the cancellation of singularities for infrared-safe observables $[4,5]$. Alternatively, one can try to tackle the infrared problem at the root, recognising that it originates from an inadequate definition of asymptotic states. This viewpoint led to the development of the method of coherent states, by means of which an infrared-finite perturbative $S$-matrix can be defined, both in QED [6] and for non-abelian theories [7]. Remarkably, the study of infrared singularities for nonabelian gauge theories continues to this day to be a very active field of research, both for theoretical reasons, and in view of phenomenological applications. On the theoretical side, a general understanding of the all-order infrared factorisation properties of non-abelian multi-particle scattering amplitudes has been developed over the years [8-24], and the relevant soft and collinear anomalous dimensions have been computed to very high orders, uncovering interesting new mathematical structures [25-38]. On the phenomenological side, a vast effort has been devoted to the construction of efficient algorithms for the subtraction of infrared singularities at high perturbative orders, in both virtual corrections and real radiation contributions to cross sections of experimental interest at colliders. In this case, the most significant problem is the treatment of unresolved real radiation for complex observables, in the presence of intricate experimental cuts. Recent advances in this direction have been reviewed in ref. [39]. 
All the developments briefly summarised above are based on standard, if advanced, techniques of quantum field theory in four dimensions, including diagrammatic tools, renormalisation group equations, Ward identities and effective field theories. In recent years, starting with refs. [40, 41], a remarkable novel formulation of the infrared problem has been introduced, based on the discovery of enhanced long-distance symmetries of theories involving massless particles. Within this framework, the infrared behaviour of scattering amplitudes is determined by infinite-dimensional asymptotic symmetries of the massless theory under consideration, acting on the 'celestial' sphere intersecting the future (or past) light cone at asymptotic distances. For gravity, these symmetries have been known for a long time [42, 43], while in the case of gauge theories they take the form of 'large' gauge transformations with non-trivial action at null infinity [44-51]. In this context, soft factorisation theorems for tree-level radiative amplitudes, and their next-to-leading-power extensions, emerge as Ward identities of these asymptotic symmetries. The first few years of work in this fast-developing field have been reviewed in [52], where one can find ample references to the literature. At the moment, much of the work done within this framework is focused on tree-level amplitudes for gauge theories and gravity (see, however, refs. [53, 54], where one-loop effects are analysed), but the connection that has been uncovered between infrared properties of $d=4$ massless field theories and $d=2$ conformal invariance on the celestial sphere is intriguing, and represents a radically innovative point of view on an old problem. An important new tool introduced in this context has been the use of Mellin transforms of scattering amplitudes $[55,56]$, which are constructed to have simple transformation properties under the conformal group acting on the celestial sphere, while from a four-dimensional viewpoint they are boost eigenstates. A rapidly growing body of literature (see, for example, refs. [57-67]) is exploring the properties of these celestial amplitudes, including detailed comparisons with known soft and collinear limits. Interestingly, related ideas have also started to be applied in a more phenomenological context [68].

In this paper, we will take a different viewpoint: instead of considering the full multiparticle scattering amplitude, we will take advantage of its factorisation properties in the infrared, and focus on the universal infrared colour operator responsible for generating all soft and collinear singularities. Colour correlations for this operator are well understood to all orders in perturbation theory, and they originate from correlators of straight, semi-infinite Wilson lines, emerging from the hard scattering and pointing in the light-like directions defined by the momenta of the hard particles participating in the scattering. The role of scale and conformal invariance in establishing the form of this infrared operator was already pointed out in refs. [18, 19], and the conformal properties of the infrared factor were instrumental in the determination of the three-loop infrared anomalous dimension by a bootstrap approach in ref. [33]. More recently, a very important observation in this direction was made in ref. [69]: examining the abelian case, Kalyanapuram noticed that the one-loop soft factor for QED-like theories is very closely connected to a correlator of vertex operators for free bosons on the celestial sphere. Here, we will pursue this remarkable connection, and propose an extension to the non-abelian case.

At the outset, such a generalisation appears to be difficult to achieve, for several reasons: first of all, in QED the exponentiation of the infrared factor is essentially one- 
loop exact (as it is in gravity [70]), while, in a non-abelian theory, intricate corrections emerge at all perturbative orders; next, as a consequence, the scale dependence of the nonabelian soft operator is non-trivial, and possibly entwined with colour correlations; finally, and more importantly, the non-abelian infrared factor is a colour operator and not just a number. These obstacles not withstanding, we will be able to propose a generalisation of the results of ref. [69] to non-abelian massless gauge theories, in terms of a conformal theory of Lie-algebra-valued free bosons on the celestial sphere.

We will begin in section 2 by providing a bird's-eye view of our current understanding of infrared divergences for massless non-abelian scattering amplitudes, focusing our attention on colour correlations, which arise exclusively from soft gauge boson exchanges. In section 3, concentrating on colour correlations with a dipole structure, which are understood to all orders, we will translate known results in terms of variables defined on the celestial sphere. Remarkably, we will find that all dependence on the gauge coupling, on the scale, and on the dimensional regulator factorises from kinematic and colour correlations, and can be collected in a single universal function which is well-known in perturbative QCD, giving, among other things, the all-order perturbative expression for the gluon Regge trajectory in high-energy scattering [71-75]; dependence on colour and kinematics is then strikingly simple, and naturally expressed in terms of distances on the celestial sphere. In section 4, we introduce a Lie-algebra-valued free-boson conformal theory on the celestial sphere. Vertex operators in this theory are colour matrices, in representations corresponding to the hard particles participating in the scattering. That notwithstanding, they have a well-defined conformal weight, and they form well-behaved correlation functions, which could be seen as colour-kinematic duals $[76,77]$ of the integrands of bosonic string amplitudes. These correlators exactly reproduce the all-order expression for infrared divergences of gauge amplitudes with dipole colour correlations.

In order to further test the correspondence between the conformal theory and the gauge theory, we note the presence in the celestial theory of a Lie-algebra-valued conserved Noether current, whose OPE with vertex operators reproduces the tree-level soft-gluon current responsible for soft real radiation in the gauge theory. This provides strong evidence for identifying this Noether current with the Kac-Moody current constructed in refs. [40, 46] by considering asymptotic limits of four-dimensional gauge fields. Further, we use the conformal OPE of vertex operators to study collinear limits of the infrared factor, and we recover the expected expression for the splitting anomalous dimension for dipole correlations $[19,78]$, once again an all-order result. In section 5, we discuss some of the many questions that our proposal leaves open at this stage. In particular, we discuss the matching between the celestial coupling and the gauge-theory coupling factor, noting that it would be of great interest to develop an understanding of the role played by scale dependence in the correspondence between the celestial and the four-dimensional theory. We emphasise that, in our view, the theory as presented here is incomplete, and we expect that a generalisation, or possibly a deformation, of the conformal theory we propose, should be able to predict, and indeed compute, the quadrupole correlations which arise in the gauge theory starting at three loops, and are explicitly known at that order [31-33]. We conclude in section 6 summarising our results and suggesting several open lines for future research. 


\section{Non-abelian soft anomalous dimensions: a primer}

The structure of infrared divergences for on-shell scattering amplitudes in massless nonabelian gauge theories is understood in remarkable generality. To summarise what is known, consider an $n$-point amplitude in dimensional regularisation, which we write as

$$
\mathcal{A}_{n}^{a_{1} \ldots a_{n}}\left(\frac{p_{i}}{\mu}, \alpha_{s}(\mu), \epsilon\right)
$$

displaying the colour indices $a_{i}$ for each external particle. Note that we are allowing for particles in different representations of the gauge group, so $a_{i}$ is not necessarily an adjoint index; furthermore, renormalisation has already been performed, and we are working in $d=4-2 \epsilon>4$, so that $\epsilon<0$, to regularise infrared singularities; finally, we are not displaying polarisation indices, which will be irrelevant in what follows. If desired, one can select a basis of colour tensors $c_{M}^{a_{1} \ldots a_{n}}$ spanning the space of allowed colour configurations for the process at hand: the amplitude in eq. (2.1) is a vector in that space, whose components in the chosen basis can be computed by suitable projections.

Infrared divergences arise from long-distance exchanges of virtual particles, and they can be shown to factorise in the form $[11,16,18,19,22,24]$

$$
\mathcal{A}_{n}\left(\frac{p_{i}}{\mu}, \alpha_{s}(\mu), \epsilon\right)=\mathcal{Z}_{n}\left(\frac{p_{i}}{\mu}, \alpha_{s}(\mu), \epsilon\right) \mathcal{H}_{n}\left(\frac{p_{i}}{\mu}, \alpha_{s}(\mu), \epsilon\right),
$$

where colour indices are understood, $\mathcal{Z}_{n}$ is a universal colour operator generating all infrared poles in $\epsilon$, and acting on a colour vector $\mathcal{H}_{n}$, which is process-dependent and finite as $\epsilon \rightarrow 0$. The divergent factor $\mathcal{Z}_{n}$ satisfies a renormalisation group equation, which can be solved in the form

$$
\mathcal{Z}_{n}\left(\frac{p_{i}}{\mu}, \alpha_{s}(\mu), \epsilon\right)=P \exp \left[\frac{1}{2} \int_{0}^{\mu^{2}} \frac{d \lambda^{2}}{\lambda^{2}} \Gamma_{n}\left(\frac{p_{i}}{\lambda}, \alpha_{s}(\lambda, \epsilon)\right)\right],
$$

where $\alpha_{s}(\lambda, \epsilon)$ is the $d$-dimensional running coupling, satisfying

$$
\lambda \frac{\partial \alpha_{s}}{\partial \lambda} \equiv \beta\left(\alpha_{s}, \epsilon\right)=-2 \epsilon \alpha_{s}-\frac{\alpha_{s}^{2}}{2 \pi} \sum_{k=0}^{\infty}\left(\frac{\alpha_{s}}{\pi}\right)^{k} b_{k} .
$$

Eq. (2.3) features the central object of our discussion, the infrared anomalous dimension matrix, which we denote by $\Gamma_{n}$. Note that the initial condition in eq. (2.3) has been fixed in the infrared, by making use of the fact that $\alpha_{s}(\lambda=0, \epsilon<0)=0$; note also that the matrix $\Gamma_{n}$ is finite as $\epsilon \rightarrow 0$, and all infrared singularities arise from the scale integration.

The matrix $\Gamma_{n}$ is clearly a fundamental object for perturbative gauge theories: for massless theories, it is fully known up to three loops for any $n$ and for any gauge group and representation content [31]. What is most remarkable, however, is the fact that its all-order structure is strongly constrained by an underlying scale invariance of the infrared sector. In order to describe this structure, it is best not to write $\Gamma_{n}$ is a specific basis, but rather to adopt the basis-independent colour-operator notation of refs. [79, 80]. In this language, one introduces, for each hard particle $i, i=1, \ldots, n$, a colour operator $\mathbf{T}_{i}$, which acts on 
the finite factor $\mathcal{H}_{n}$ as a generator of the gauge algebra in the appropriate representation. ${ }^{1}$ The colour operators $\mathbf{T}_{i}$ carry an adjoint index, and satisfy

$$
\left[\mathbf{T}_{i}^{a}, \mathbf{T}_{i}^{b}\right]=\mathrm{i} f_{c}^{a b} \mathbf{T}_{i}^{c}, \quad \mathbf{T}_{i} \cdot \mathbf{T}_{i} \equiv \mathbf{T}_{i}^{a} \mathbf{T}_{i}^{b} \delta_{a b}=C_{i}^{(2)}, \quad \sum_{i=1}^{n} \mathbf{T}_{i}=0
$$

where $C_{i}^{(2)}$ is the quadratic Casimir eigenvalue of the gauge algebra in the representation of particle $i$, while the last equation enforces colour conservation, and must be interpreted as constraint on the action of the colour operators $\mathbf{T}_{i}$ on the finite factor $\mathcal{H}_{n}$, dictated by gauge invariance. Note that colour operators associated with different hard particles commute, since $\mathcal{Z}_{n}$ is an operator in the tensor product of the colour representations of the $n$ particles, and each $\mathbf{T}_{i}$ acts on a single factor in that product.

Let us now discuss the all-order structure of the infrared anomalous dimension matrix. As we will see, a crucial ingredient is the light-like cusp anomalous dimension $\gamma_{K, r}\left(\alpha_{s}\right)$, governing the UV divergences of form factors of Wilson lines in representation $r$ [81-85]. For the sake of simplicity, we will consider the approximation in which the cusp anomalous dimension satisfies 'Casimir scaling', i.e. we will write

$$
\gamma_{K, r}\left(\alpha_{s}\right)=C_{r}^{(2)} \widehat{\gamma}_{K}\left(\alpha_{s}\right),
$$

where $\widehat{\gamma}_{K}\left(\alpha_{s}\right)$ is representation-independent. This approximation is known to fail at the four-loop level [34, 35, 86-89], where contributions proportional to quartic Casimir eigenvalues have been shown to appear, with expected consequences on the structure of $\Gamma_{n}$ at four loops and beyond [90, 91]. With this single simplifying approximation, the soft anomalous dimension matrix admits the all-order representation

$$
\Gamma_{n}\left(\frac{p_{i}}{\mu}, \alpha_{s}(\mu)\right)=\Gamma_{n}^{\text {dipole }}\left(\frac{s_{i j}}{\mu^{2}}, \alpha_{s}(\mu)\right)+\Delta_{n}\left(\rho_{i j k l}, \alpha_{s}(\mu)\right) .
$$

The colour-dipole term $\Gamma_{n}^{\text {dipole }}$ is the only contribution at one and two loops, and can be written as

$$
\Gamma_{n}^{\operatorname{dipole}}\left(\frac{s_{i j}}{\mu^{2}}, \alpha_{s}(\mu)\right)=\frac{1}{2} \widehat{\gamma}_{K}\left(\alpha_{s}(\mu)\right) \sum_{i=1}^{n} \sum_{j=i+1}^{n} \log \frac{-s_{i j}+\mathrm{i} \eta}{\mu^{2}} \mathbf{T}_{i} \cdot \mathbf{T}_{j}-\sum_{i=1}^{n} \gamma_{i}\left(\alpha_{s}(\mu)\right),
$$

where we assumed for simplicity that all particles are outgoing, $s_{i j}=2 p_{i} \cdot p_{j}$, and $\gamma_{i}\left(\alpha_{s}\right)$ is the UV anomalous dimensions for the field corresponding to particle $i$. The conformal correction $\Delta_{n}$ arises starting at three loops, with at least four particles, and it is constrained by scale invariance to depend only upon the conformal cross-ratios

$$
\rho_{i j k l}=\frac{p_{i} \cdot p_{j} p_{k} \cdot p_{l}}{p_{i} \cdot p_{l} p_{j} \cdot p_{k}}=\frac{s_{i j} s_{k l}}{s_{i l} s_{j k}} .
$$

At the three-loop level, $\Delta_{n}$ was computed in refs. [31-33], and it is built out of quadrupole correlations of the form

$$
F_{i j k l}(\{\rho\}) f_{a b e} f_{c d}{ }^{e} \mathbf{T}_{i}^{a} \mathbf{T}_{j}^{a} \mathbf{T}_{k}^{c} \mathbf{T}_{l}^{d},
$$

\footnotetext{
${ }^{1}$ Thus, for example, for an outgoing quark one has $\mathbf{T}_{i} \rightarrow T_{i j}^{a}$, where $T^{a}$ is a generator in the fundamental representation of $\mathrm{SU}(N)$, while for a gluon one has $\mathbf{T}_{i} \rightarrow-\mathrm{i} f_{c d}^{a}$, where $f_{c d}^{a}$ are the structure constants.
} 
where the kinematic dependence is contained in the functions $F_{i j k l}$; by Bose symmetry and colour conservation, actually only one function is involved, and it turns out to be an extremely simple combination of weight- 5 single-valued harmonic polylogarithms [92, 93].

In the present context, it is important to note that the dipole term can be organised in a more useful way by exploiting colour conservation to simplify the scale dependence, which will need to be integrated. One may write

$$
\begin{aligned}
\Gamma_{n}^{\text {dipole }}\left(\frac{s_{i j}}{\lambda^{2}}, \alpha_{s}(\lambda, \epsilon)\right)= & \frac{1}{2} \widehat{\gamma}_{K}\left(\alpha_{s}(\lambda, \epsilon)\right) \sum_{i=1}^{n} \sum_{j=i+1}^{n} \ln \left(\frac{-s_{i j}+\mathrm{i} \eta}{\mu^{2}}\right) \mathbf{T}_{i} \cdot \mathbf{T}_{j} \\
& -\sum_{i=1}^{n} \gamma_{i}\left(\alpha_{s}(\lambda, \epsilon)\right)-\frac{1}{4} \widehat{\gamma}_{K}\left(\alpha_{s}(\lambda, \epsilon)\right) \ln \left(\frac{\mu^{2}}{\lambda^{2}}\right) \sum_{i=1}^{n} C_{i}^{(2)} \\
\equiv & \Gamma_{n}^{\text {corr. }}\left(\frac{s_{i j}}{\mu^{2}}, \alpha_{s}(\lambda, \epsilon)\right)+\Gamma_{n}^{\text {singl. }}\left(\frac{\mu^{2}}{\lambda^{2}}, \alpha_{s}(\lambda, \epsilon)\right)
\end{aligned}
$$

where $\mu$ is a fixed scale, while $\lambda$ is the integration variable in eq. (2.3). In this form, one can show that (aside from poles originating from the running of the coupling) the first term, which contains dipole colour correlations, generates only single poles of soft origin, while the remaining terms, which are colour singlets, generate single poles of hard-collinear origin, as well as double poles of soft-collinear origin. In order to compute them explicitly, one may use the explicit expression for the $d$-dimensional running coupling at the desired order, and perform the scale integration in eq. (2.3). For example, at the one-loop level, one may use the lowest-order expression for $d$-dimensional running coupling

$$
\alpha_{s}(\lambda, \epsilon)=\alpha_{s}(\mu)\left(\frac{\lambda^{2}}{\mu^{2}}\right)^{-\epsilon},
$$

together with the basic integrals

$$
\int_{0}^{\mu^{2}} \frac{d \lambda^{2}}{\lambda^{2}} \alpha_{s}(\lambda, \epsilon)=-\frac{1}{\epsilon} \alpha_{s}(\mu), \quad \int_{0}^{\mu^{2}} \frac{d \lambda^{2}}{\lambda^{2}} \ln \left(\frac{\lambda^{2}}{\mu^{2}}\right) \alpha_{s}(\lambda, \epsilon)=-\frac{1}{\epsilon^{2}} \alpha_{s}(\mu), \quad(\epsilon<0) .
$$

Note that in a gauge theory which is conformal in $d=4$, such as $\mathcal{N}=4$ Super-Yang-Mills theory, the result in eq. (2.12) is exact, and indeed the logarithm of the infrared operator $\mathcal{Z}_{n}$ has only single and double poles [94], which are trivially determined in terms of the perturbative coefficients of the singlet anomalous dimensions $\widehat{\gamma}_{K}$ and $\gamma_{i}$. In what follows, we will concentrate on the colour correlated term, $\Gamma_{n}^{\text {corr. }}$. It is useful to remember that it originates from the Wilson-line correlator

$$
\mathcal{S}_{n}\left(\beta_{i} \cdot \beta_{j}, \alpha_{s}(\mu), \epsilon\right) \equiv\left\langle 0\left|\prod_{i=1}^{n} \Phi_{\beta_{i}}(\infty, 0)\right| 0\right\rangle,
$$

where $\beta_{i}$ are dimensionless four-velocity vectors parallel to the momenta $p_{i}$, and $\Phi_{\beta_{i}}$ are semi-infinite Wilson lines along the classical trajectories of the particles exiting the hard interaction vertex

$$
\Phi_{\beta}(\infty, 0) \equiv P \exp \left[\mathrm{i} g \int_{0}^{\infty} d \lambda \beta \cdot A(\lambda \beta)\right] .
$$


Note that the Wilson lines in eq. (2.14) have open colour indices at both ends, so that $\mathcal{S}_{n}$, as announced, is a colour operator acting on the hard factor $\mathcal{H}_{n}$ in eq. (2.2). Classically, the correlator in eq. (2.14) is invariant under the rescalings $\beta_{i} \rightarrow \kappa_{i} \beta_{i}$, however this invariance is broken by collinear divergences in the massless case: the dipole contribution to the anomalous dimension matrix arises as a solution to the corresponding 'anomaly equation', where the 'anomaly' is expressed by the cusp anomalous dimension [18, 19].

\section{Colour correlations on the celestial sphere}

Taking inspiration from refs. [33, 69], we now rewrite the colour-correlated part of the infrared anomalous dimension matrix in terms of coordinates on the celestial sphere. We parametrise the momenta $p_{i}$ as

$$
p_{i}^{\mu}=\omega_{i}\left\{1+z_{i} \bar{z}_{i}, z_{i}+\bar{z}_{i},-\mathrm{i}\left(z_{i}-\bar{z}_{i}\right), 1-z_{i} \bar{z}_{i}\right\},
$$

which is real if (as we assume) $\bar{z}_{i}=z_{i}^{*}$, and implies

$$
s_{i j}=2 p_{i} \cdot p_{j}=4 \omega_{i} \omega_{j}\left|z_{i}-z_{j}\right|^{2},
$$

so that all momenta are indeed light-like, $p_{i}^{2}=0$. We can define the four-velocities $\beta_{i}$, for example, by $p_{i}^{\mu}=\sqrt{2} \omega_{i} \beta_{i}^{\mu}$, so that $\beta_{i} \cdot \beta_{j}=\left|z_{i}-z_{j}\right|^{2}$. Then we notice that (as expected from eq. (2.14)) the energies $w_{i}$ do not contribute to colour correlations, again as an effect of colour conservation. Indeed one can use

$$
\log \left(-s_{i j}+\mathrm{i} \eta\right)=\log \left(\left|z_{i}-z_{j}\right|^{2}\right)+\log \omega_{i}+\log \omega_{j}+2 \log 2+\mathrm{i} \pi,
$$

and perform the colour sum on terms that do not depend simultaneously on $i$ and $j$. All these terms can then be shuffled into the colour-singlet contribution to $\Gamma_{n}$, so that we can write

$$
\Gamma_{n}^{\text {dipole }}\left(\frac{s_{i j}}{\lambda^{2}}, \alpha_{s}(\lambda, \epsilon)\right) \equiv \widehat{\Gamma}_{n}^{\text {corr. }}\left(z_{i j}, \alpha_{s}(\lambda, \epsilon)\right)+\widehat{\Gamma}_{n}^{\text {singl. }}\left(\frac{\omega_{i}}{\lambda}, \alpha_{s}(\lambda, \epsilon)\right)
$$

where we defined $z_{i j} \equiv z_{i}-z_{j}$. The colour-singlet contribution is given by

$$
\widehat{\Gamma}_{n}^{\text {singl. }}\left(\frac{\omega_{i}}{\lambda}, \alpha_{s}(\lambda, \epsilon)\right)=-\sum_{i=1}^{n} \gamma_{i}\left(\alpha_{s}(\lambda, \epsilon)\right)-\frac{1}{4} \widehat{\gamma}_{K}\left(\alpha_{s}(\lambda, \epsilon)\right) \sum_{i=1}^{n} \ln \left(\frac{-4 \omega_{i}^{2}+\mathrm{i} \eta}{\lambda^{2}}\right) C_{i}^{(2)}
$$

while

$$
\widehat{\Gamma}_{n}^{\text {corr. }}\left(z_{i j}, \alpha_{s}(\lambda, \epsilon)\right)=\frac{1}{2} \widehat{\gamma}_{K}\left(\alpha_{s}(\lambda, \epsilon)\right) \sum_{i=1}^{n} \sum_{j=i+1}^{n} \ln \left(\left|z_{i j}\right|^{2}\right) \mathbf{T}_{i} \cdot \mathbf{T}_{j} .
$$

The remarkable fact about eq. (3.6) is that the scale dependence is factorised, universal, and free from colour correlations. This enables us to write the colour-correlated part of the infrared operator $\mathcal{Z}_{n}$ in a strikingly simple way, as

$$
\begin{aligned}
\mathcal{Z}_{n}^{\text {corr. }}\left(z_{i j}, \alpha_{s}(\mu), \epsilon\right) & \equiv \exp \left[\int_{0}^{\mu} \frac{d \lambda}{\lambda} \widehat{\Gamma}_{n}^{\text {corr. }}\left(z_{i j}, \alpha_{s}(\lambda, \epsilon)\right)\right] \\
& =\exp \left[-K\left(\alpha_{s}(\mu), \epsilon\right) \sum_{i=1}^{n} \sum_{j=i+1}^{n} \ln \left(\left|z_{i j}\right|^{2}\right) \mathbf{T}_{i} \cdot \mathbf{T}_{j}\right]
\end{aligned}
$$


where the universal scale-dependent prefactor is given by

$$
K\left(\alpha_{s}(\mu), \epsilon\right)=-\frac{1}{2} \int_{0}^{\mu} \frac{d \lambda}{\lambda} \widehat{\gamma}_{K}\left(\alpha_{s}(\lambda, \epsilon)\right) .
$$

The function $K$ is well-known in QCD, where it plays a role in a number of different settings: importantly, it defines the perturbative Regge trajectory in the high-energy limit of $2 \rightarrow 2$ scattering amplitudes [71-75], and it computes soft-collinear poles for Sudakov form factors $[13,95]$. In a gauge theory with conformal symmetry in $d=4$ one finds

$$
K\left(\alpha_{s}, \epsilon\right)=\sum_{n=1}^{\infty}\left(\frac{\alpha_{s}}{\pi}\right)^{n} \frac{\widehat{\gamma}_{K}^{(n)}}{4 n \epsilon},
$$

where $\widehat{\gamma}_{K}^{(n)}$ are the coefficients of the expansion of the function $\widehat{\gamma}_{K}\left(\alpha_{s}\right)$ in powers of $\alpha_{s} / \pi$. With a non-vanishing $\beta$ function one finds instead [96]

$$
\begin{aligned}
K\left(\alpha_{s}, \epsilon\right)= & \frac{\alpha_{s}}{\pi} \frac{\widehat{\gamma}_{K}^{(1)}}{4 \epsilon}+\left(\frac{\alpha_{s}}{\pi}\right)^{2}\left(\frac{\widehat{\gamma}_{K}^{(2)}}{8 \epsilon}+\frac{b_{0} \widehat{\gamma}_{K}^{(1)}}{32 \epsilon^{2}}\right) \\
& +\left(\frac{\alpha_{s}}{\pi}\right)^{3}\left(\frac{\widehat{\gamma}_{K}^{(3)}}{12 \epsilon}+\frac{b_{0} \widehat{\gamma}_{K}^{(2)}+b_{1} \widehat{\gamma}_{K}^{(1)}}{48 \epsilon^{2}}+\frac{b_{0}^{2} \widehat{\gamma}_{K}^{(1)}}{192 \epsilon^{3}}\right)+\mathcal{O}\left(\alpha_{s}^{4}\right),
\end{aligned}
$$

and all the higher-order coefficients can be explicitly determined [95] in terms of $b_{n}$ and $\widehat{\gamma}_{K}^{(n)}$. A simple factorised form for the infrared operator $\mathcal{Z}_{n}$ in terms of the function $K$ had previously been observed in the high-energy limit [72-75], but the complete generality of eq. (3.7) only becomes apparent in the celestial coordinates of eq. (3.1).

As noted in ref. [69] in the abelian case (where the operators $\mathbf{T}_{i}$ are replaced by the electric charges $e_{i}$, and the one-loop approximation to the cusp anomalous dimension suffices), eq. (3.7) bears a striking resemblance to a correlator of vertex operators in a $d=2$ conformal theory. In the non-abelian case, we need to handle the matrix structure of eq. (3.7), but the eventual connection to conformal invariance is actually strengthened by the knowledge that high-order corrections depend only on conformal cross-ratios, which in this language read $\rho_{i j k l}=\left|z_{i j}\right|^{2}\left|z_{k l}\right|^{2} /\left(\left|z_{i l}\right|^{2}\left|z_{k j}\right|^{2}\right)$. In what follows, we will make a proposal for the conformal theory generating eq. (3.7), and speculate about the origin of higher-order corrections.

\section{A theory of Lie-algebra-valued free bosons}

We consider a set of scalar fields on a two-dimensional sphere, $\phi_{a}(z, \bar{z})$, forming a multiplet in the adjoint representation of a Lie algebra, which we will take to be $s u\left(N_{c}\right)$, so that $a=1, \ldots, N_{c}^{2}-1$. For these fields one can naturally choose the action

$$
S(\phi)=\frac{1}{2 \pi} \int d^{2} z \partial_{z} \phi^{a}(z, \bar{z}) \partial_{\bar{z}} \phi_{a}(z, \bar{z}),
$$

so that we have a theory of Lie-algebra-valued free bosons. Since we will have to pair these free bosons with colour operators, one may also note from the outset that the scalars could be naturally organised into a matrix field

$$
\Phi_{r}(z, \bar{z}) \equiv \phi_{a}(z, \bar{z}) T_{r, z}^{a},
$$


where $T_{r, z}^{a}$ are generators of the group in the irreducible representation $r$. Considering the gauge-theory structure we are trying to mimic, the generators must be associated with the point $z$ on the sphere where the fields are evaluated: in particular, this means that the colour-space commutator $\left[\Phi\left(z_{1}, \bar{z}_{1}\right), \Phi\left(z_{2}, \bar{z}_{2}\right)\right]$ must vanish, so long as $z_{1} \neq z_{2}$. Products of matrix fields at different points are operators on the tensor product of the corresponding irreducible representations, and indeed one is free to consider products of matrix fields belonging to different representations. In terms of these matrix fields, one can write the action in eq. (4.1) as

$$
S(\Phi)=\frac{1}{2 \pi t_{r}} \int d^{2} z \operatorname{Tr}\left[\partial_{z} \Phi_{r}(z, \bar{z}) \partial_{\bar{z}} \Phi_{r}(z, \bar{z})\right]
$$

where we normalised the generators according to $\operatorname{Tr}\left(T_{r}^{a} T_{r}^{b}\right)=t_{r} \delta^{a b}$. Writing the action in the form of eq. (4.3), with matrices $T_{r, z}^{a}$ that are taken to depend on $z$, suggests that the proper underlying mathematical structure should be a gauge bundle on the sphere, and one might then expect that the derivative should be replaced by a covariant derivative. Such an extension is non-trivial for at least two reasons: first of all, one would need a precise form for the gauge connection on the celestial sphere; next, we emphasise that the collinear limits where two punctures $z_{i}$ and $z_{j}$ coincide is singular in our framework: indeed, eq. (2.2), which was our starting point, was derived for fixed-angle scattering amplitudes, where none of the invariants $s_{i j}$ vanishes. In any case, we expect that corrections to eq. (4.3) arising from this issue would be of higher order in the gauge coupling, and furthermore they would contribute only to colour structures beyond dipoles, involving the structure constants $f_{a b c}$. The free action in eq. (4.1) will therefore suffice for our present purposes, and we will take it as the definition of the theory. We will briefly come back to this issue in section 5 .

It is clear that in this setup the nature of the fields $\phi^{a}(z, \bar{z})$ as fields in a conformal theory on the sphere is quite decoupled from the matrix structure that we have superimposed. Indeed, eq. (4.1) is essentially the bosonic string action in a conformal gauge, with scalar fields interpreted as coordinates in a Lie algebra rather than a spacetime: one could describe it as a 'single copy' of the tree-level bosonic string, in the spirit of colour-kinematic duality [76]. Most of the well-known formalism for the treatment of two-dimensional free scalar fields [97] is then simply inherited by our theory with only minor changes. In particular, the equations of motion are

$$
\partial_{z} \partial_{\bar{z}} \phi^{a}(z, \bar{z})=0
$$

implying that the field $\partial_{z} \phi^{a}$ is holomorphic, while $\partial_{\bar{z}} \phi^{a}$ is anti-holomorphic. In the quantum theory, when taking matrix elements of products of fields, eq. (4.4) leads to

$$
\partial_{z} \partial_{\bar{z}} \phi^{a}(z, \bar{z}) \phi^{b}(w, \bar{w})=-\pi \delta^{a b} \delta^{2}(z-w, \bar{z}-\bar{w}),
$$

which suggests the definition of the normal-ordered product

$$
: \phi^{a}(z, \bar{z}) \phi^{b}(w, \bar{w}):=\phi^{a}(z, \bar{z}) \phi^{b}(w, \bar{w})+\frac{1}{2} \delta^{a b} \log |z-w|^{2}
$$


whose derivatives are free of contact terms. Following standard arguments, the theory defined by eq. (4.1) has a traceless conserved energy-momentum tensor, which can be organised into holomorphic and anti-holomorphic components, with

$$
T(z)=-: \partial_{z} \phi^{a}(z, \bar{z}) \partial_{z} \phi_{a}(z, \bar{z}):, \quad \widetilde{T}(\bar{z})=-: \partial_{\bar{z}} \phi^{a}(z, \bar{z}) \partial_{\bar{z}} \phi_{a}(z, \bar{z}): .
$$

More interestingly, since the action in eq. (4.1) involves only derivatives of $\phi^{a}$, there is a symmetry under translations in field space, which are now to be interpreted as translations in the Lie algebra. The Noether current for this symmetry is Lie-algebra valued, and we will propose that it should be interpreted as a leading-order contribution to a Kac-Moody current for the full theory, to be identified with the current constructed in $[40,46]$. Its holomorphic and anti-holomorphic components are simply given by the derivatives of the scalar fields

$$
j^{a}(z)=\partial_{z} \phi^{a}(z, \bar{z}), \quad \tilde{j}^{a}(\bar{z})=\partial_{\bar{z}} \phi^{a}(z, \bar{z}) .
$$

Following the standard lore for free bosons, we may now introduce vertex operators for this theory, in the form

$$
V(z, \bar{z}) \equiv: \mathrm{e}^{\mathrm{i} \kappa \mathbf{T}_{z} \cdot \phi(z, \bar{z})}:=: \mathrm{e}^{\mathrm{i} \kappa \Phi(z, \bar{z})}:,
$$

where we dropped the representation label $r$, and we introduced the operator notation discussed in section 2. The vertex operators in eq. (4.9) are matrices in the selected representation of the gauge group: one can think of them as operators defined on the celestial sphere and acting on the bulk colour degrees of freedom. We have introduced a coupling $\kappa$ in the exponent, allowing for the fact that the normalisation of the fields at this stage is arbitrary, and we will need to match our results to the gauge theory. The vertex operators in eq. (4.9) are reminiscent of those used in the vertex-operator construction of Kac-Moody algebras [98], but we emphasise that they are quite different. In the KacMoody construction, one works in the Cartan-Weyl basis for the Lie algebra, and one introduces a (Fubini-Veneziano) scalar field $Q_{i}(z, \bar{z})$ only for generators $H_{i}$ in the Cartan subalgebra; one then builds a vertex operator for every root $\alpha$ of the algebra, with an exponent proportional to $\alpha^{i} Q_{i}$. These operators build a representation of the Kac-Moody algebra in the Hilbert space of the conformal theory, but they act as numbers in colour space. In our case, on the other hand, in order to reproduce the gauge theory results, we need an expression treating all colour degrees of freedom on the same footing, which results in colour matrices. Pursuing an alternative analogy, one may note that a standard construction of Kac-Moody algebras from ordinary Lie algebras involves promoting the parameters $\theta^{a}$ of the Lie algebra to functions $\theta^{a}(z)$ on a circle $S^{1}$, and taking Fourier modes: in a sense, eq. (4.9) is similar, with the circle replaced by the sphere $S^{2}$. Finally, we must note a formal analogy between the fields $\phi^{a}$ in eq. (4.9) and the interpolating fields for Reggeized gluons introduced in ref. [99], where however the gauge-theory Wilson lines are not semi-infinite but infinite, and the sphere is replaced by the transverse plane. For the purposes of the present paper, we will simply take eq. (4.9) at face value, and derive its properties.

With the operators in eq. (4.9), we can construct correlation functions which bear a direct analogy with the world-sheet integrands of tree-level bosonic string amplitudes. 
There are a number of potential obstacles for the consistency of this procedure, that we will now consider, but, interestingly, they can all be bypassed using well-understood properties of the operators $\mathbf{T}_{z}$.

- First of all, we need to establish that the vertex operator in eq. (4.9) is a good conformal field. This appears far from obvious given its matrix structure. Once again, following standard procedures, one can write down an explicit solution of the equations of motions for the fields $\phi_{a}(z, \bar{z})$ in terms of a Fourier expansion, and proceed to quantise the theory by interpreting the coefficients of the Fourier modes as (rescaled) harmonic-oscillator creation and annihilation operators. In this formalism, the conformal dimension $h(=\bar{h})$ of the vertex operator emerges entirely from the normal ordering, bringing annihilation operators to the left and creation operators to the right. The colour operators $\mathbf{T}_{z}$ are spectators in this calculation, and the standard free-field ('bosonic string') result is reproduced. Pursuing the analogy, we recall that in a string-theory context one finds

$$
V_{\text {c.s. }}(z, \bar{z}) \equiv: \mathrm{e}^{\mathrm{i} k^{\mu} X_{\mu}(z, \bar{z})}: \quad \longrightarrow \quad h=\frac{1}{4} k^{\mu} k^{\nu} \eta_{\mu \nu}=\frac{k^{2}}{4},
$$

where the space-time metric $\eta_{\mu \nu}$ comes from the interpretation of the fields $X^{\mu}$ as space-time coordinates. In the case of eq. (4.9), the fields $\phi_{a}$ are Lie-algebra coordinates, and the metric is simply the Cartan-Killing metric $\delta_{a b}$. Therefore

$$
V(z, \bar{z}) \equiv: \mathrm{e}^{\mathrm{i} \kappa \mathbf{T}_{z} \cdot \phi(z, \bar{z})}: \quad \longrightarrow \quad h=\frac{\kappa^{2}}{4} \mathbf{T}_{z} \cdot \mathbf{T}_{z}=\frac{\kappa^{2}}{4} C_{r}^{(2)},
$$

where $C_{r}^{(2)}$ is the quadratic Casimir eigenvalue for representation $r$, which, crucially, is a number. In a conformal theory, this result must be consistent with the scaling properties of two-point functions of vertex operators, which must satisfy

$$
\left\langle V\left(z_{1}, \bar{z}_{1}\right) V\left(z_{2}, \bar{z}_{2}\right)\right\rangle \sim\left|z_{12}\right|^{-2 \Delta},
$$

with $\Delta=h+\bar{h}$. As we will see below, this is indeed the case, upon enforcing colour conservation, which for the two-point function requires $\mathbf{T}_{1}=-\mathbf{T}_{2}$.

- Of course, the analogy with strings should not be pushed too far: for example, we note that, in the case of a string theory, the value of $k^{2}$ in eq. (4.10) is fixed, and allowed values for different vertex operators give the mass spectrum of the string. The origin of the constraint, however, is the fact that string-theory vertex operators relevant to string amplitudes are integrated over the world sheet, to preserve diffeomorphism invariance. Invariance under rescalings of the world-sheet coordinates then imposes $h=1$ for the ('tachyon') vertex operator we are considering, and integer values of $h$ for vertex operators involving prefactors with derivatives of the fields. In our case, we will never need to integrate over the locations of the punctures, which represent the (fixed) momenta of the hard particles, so there are no constraints of this kind on $h$. We are genuinely interested in the conformal correlation function in a fixed coordinate system. 
- We will be evaluating correlation functions of $n$ vertex operators of the form of eq. (4.9). In the case of bosonic strings, arguments from holomorphicity, or just the evaluation of such correlators from the path integral on the sphere [100], show that the results are consistent and scale-invariant only if momentum is conserved, $\sum_{i} k_{i}^{\mu}=0$. For string amplitudes, this has an obvious physical interpretation. In the present case, colour conservation takes the place of momentum conservation, as noted in section 2: the condition $\sum_{i} \mathbf{T}_{z_{i}}=0$, which must be satisfied when the correlator is regarded as an operator acting on the 'bulk' gauge theory, guarantees the proper scale-invariant behaviour. Correlators will be operators with support only on colour-conserving quantities, consistently with the gauge-invariance of the four-dimensional theory.

Having established that we have a bona fide conformal field theory on the sphere, and wellbehaved - though matrix-valued - vertex operators, the obvious next step is to study the correlator

$$
\mathcal{C}_{n}\left(\left\{z_{i}\right\}, \kappa\right) \equiv\left\langle\prod_{i=1}^{n} V\left(z_{i}, \bar{z}_{i}\right)\right\rangle .
$$

The evaluation of the correlator $\mathcal{C}_{n}$ is a textbook exercise (see for example chapter 6 in ref. [100]): in a conformal invariant theory, one is always allowed to use a locally flat metric (as we are doing), and one simply gets an expression analogous to the integrand of the Virasoro-Shapiro amplitude for tree-level closed strings. We find

$$
\mathcal{C}_{n}\left(\left\{z_{i}\right\}, \kappa\right)=C\left(N_{c}\right) \exp \left[\frac{\kappa^{2}}{2} \sum_{i=1}^{n} \sum_{j=i+1}^{n} \ln \left(\left|z_{i j}\right|^{2}\right) \mathbf{T}_{i} \cdot \mathbf{T}_{j}\right]
$$

where $C\left(N_{c}\right)$ is a constant dependent on the Lie algebra. This is of course the result we have been working to achieve: it is precisely of the form of eq. (3.7), which in turn generates all infrared singularities with a colour-dipole structure for any massless gauge theory and to any perturbative order. It is therefore tempting at this stage to simply state that we can set the coupling $\kappa$ to reproduce eq. (3.7) exactly. This is certainly possible, but we note at the outset that this identification raises interesting dynamical questions, concerning both the scale dependence of the answer, and dependence on the dimensional regulator $\epsilon$. We discuss this issue and some of the many important open questions in section 5. A further observation on eq. (4.14) is warranted: if one computes the correlator in eq. (4.13) by means of a path integral on a curved two-dimensional surface, as done for example in ref. [100], eq. (4.14) is modified by an extra factor arising from the Weyl anomaly. This factor cancels in the conformal theory, but would be present, for example, if one were to break scale invariance by considering a fixed sphere of finite radius $R$, thus introducing a length scale. The Weyl factor for the correlator in eq. (4.13) takes the form

$$
\mathcal{W}_{n}\left(\left\{z_{i}\right\}, \kappa\right)=\exp \left[-\frac{1}{2} \sum_{i=1}^{n} C_{i}^{(2)} g\left(z_{i}, \bar{z}_{i}\right)\right]
$$

where $g\left(z_{i}, \bar{z}_{i}\right)$ is a scale factor. The interesting point about eq. (4.15) is that the Weyl factor matches the factor arising in the gauge theory from the energy-sensitive terms that 
we have shuffled to the colour-singlet part of the anomalous dimension, $\widehat{\Gamma}_{n}^{\text {singl. }}$ in eq. (3.5). Such 'diagonal' contributions to the infrared operator $\mathcal{Z}_{n}$, with an exponent proportional to a sum of terms associated with single points $z_{i}$, cannot be expected to emerge from the celestial CFT: on the gauge theory side, they are related to collinear divergences, and do not contribute to colour correlations, but they are sensitive to particle energies.

There are further significant tests of the correspondence we have found between the infrared sector of non-abelian gauge theories and the proposed conformal theory on the celestial sphere, arising from soft and collinear limits. On the gauge-theory side, it is wellknown that the tree-level emission of a soft gluon factorises [79] from hard $n$-point scattering amplitudes (and similarly from correlators of $n$ Wilson-lines such as $\mathcal{S}_{n}$ in eq. (2.14)), and the soft factor is given by the soft current for the emission of a gluon of momentum $k$

$$
\mathbf{J}^{\mu}(k)=g \sum_{i=1}^{n} \mathbf{T}_{i} \frac{\beta_{i}^{\mu}}{\beta_{i} \cdot k}
$$

exposing the singular behaviour of the radiative amplitude as the gluon energy vanishes, as well as a set of collinear singularities as $k$ becomes parallel to $\beta_{i}$. The current is gaugeinvariant, in the sense that its longitudinal component vanishes, since

$$
k \cdot \mathbf{J}^{\mu}(k)=g \sum_{i=1}^{n} \mathbf{T}_{i}=0,
$$

by colour conservation. Remarkably, this factorisation theorem for soft non-abelian radiation can be derived in the conformal theory on the sphere, by consider a generalised correlator of vertex operators, including an insertion of the Noether current in eq. (4.8). Taking the OPE of the current with the vertex operators yields the result

$$
\left\langle\partial_{z} \phi^{a}(z, \bar{z}) \prod_{i=1}^{n} V\left(z_{i}, \bar{z}_{i}\right)\right\rangle \simeq-\frac{\mathrm{i}}{2} \sum_{i=1}^{n} \frac{\mathbf{T}_{i}^{a}}{z-z_{i}} \mathcal{C}_{n}\left(\left\{z_{i}\right\}, \kappa\right)
$$

As discussed in detail in ref. [46] (see also refs. [40, 50, 51, 61]), the r.h.s. of eq. (4.18) is the representation of the soft-gluon theorem [79] on the celestial sphere, and the poles as $z \rightarrow z_{i}$ are collinear poles. As noted above, in the gauge theory this pole is superimposed to an angle-independent soft singularity arising when the energy of the soft gluon becomes vanishingly small; the soft singularity is absent in eq. (4.18), which can be understood in the present context by recalling that soft-collinear singularities are color-uncorrelated, as shown in section 2 for virtual corrections. Colour conservation emerges in eq. (4.18) from the request that the Noether current be holomorphic as $z \rightarrow \infty$ : the correlator in eq. (4.18) must then vanish as $z^{-2}$ for large $z$, which indeed requires that $\sum_{i} \mathbf{T}_{i}=0$. Importantly, ref. [46] finds the same result by employing a current constructed in terms of asymptotic expressions for the four-dimensional fields: this provides clear evidence that the two currents are different representations of the same object, at least to the accuracy of the present calculation. In the framework of ref. [46], the soft pole is absent because the current is constructed out of field strengths rather than gauge potentials, providing an extra power of the energy in the numerator. We note that one finds an equivalent result using 
the anti-holomorphic current in eq. (4.8): ref. [46], examining double soft emission, argues that one of the two currents generates a Kac-Moody symmetry, but there are subtleties in attempting to extend this to both currents, related to the ordering of soft limits in the double-emission matrix element. An analysis of this ambiguity goes beyond the approximation we have taken so far (of including only colour-dipole correlations), since in QCD the difference between soft orderings is proportional to colour correlations involving the structure constants $f_{a b c}$, but this is without doubt a very interesting topic for future work.

Next, we turn to collinear limits, when the momenta of two hard particles become proportional, and consequently two of the punctures on the celestial sphere become close. As we noted, strict collinear limits, where some of the Mandelstam invariants $s_{i j}$ vanish, violate the factorisation theorem in eq. (2.2), and therefore lie outside the reach of our discussion. ${ }^{2}$ We can, however, study the approach to the collinear limit, when two particles $i$ and $j$ are nearly aligned, so that the invariant $s_{i j}$ is much smaller than all other invariants. In this limit, the factorisation in eq. (2.2) correctly captures all logarithms of the small invariant that are multiplied by infrared poles. With this understanding, on the gauge theory side, collinear limits of eq. (2.8) are well understood, and tested up to three loops (where of course the correction $\Delta_{n}$ must also be taken into account). On the celestial side of the correspondence, collinear limits must correspond to short distance limits, $\left|z_{i j}\right| \rightarrow 0$, and therefore can be probed by considering the OPE of the vertex operators in eq. (4.9). In order to clarify the correspondence, let us begin by giving a precise definition of the collinear limit (see also ref. [65]). Following standard practices in QCD, in order to describe the situation in which two light-like momenta $p_{1}$ and $p_{2}$ become collinear along a light-like direction $p$, we introduce the Sudakov parametrisation

$$
\begin{aligned}
& p_{1}^{\mu}=x p^{\mu}+p_{\perp}^{\mu}-\frac{p_{\perp}^{2}}{2 x p \cdot n} n^{\mu} \\
& p_{2}^{\mu}=(1-x) p^{\mu}-p_{\perp}^{\mu}-\frac{p_{\perp}^{2}}{2(1-x) p \cdot n} n^{\mu}
\end{aligned}
$$

where $n^{\mu}$ is a reference light-like vector satisying $n^{2}=n \cdot p_{\perp}=0$, and $p_{\perp}^{\mu}$ is a space-like vector orthogonal to the collinear direction, $p \cdot p_{\perp}=0$. The collinear limit is parametrised by taking $p_{\perp}^{2} \rightarrow 0$, and, in the limit, $p_{1}+p_{2}=p$. It is not difficult to translate the Sudakov parametrisation to the celestial sphere: using eq. (3.1) for the momenta $p_{1}$ and $p_{2}$, and picking a fixed vector $n^{\mu}$, for example, as

$$
n^{\mu}=\frac{1}{2}\{1,0,0,-1\} \quad \rightarrow \quad n \cdot p_{i}=\omega_{i}, \quad n \cdot p=\omega_{1}+\omega_{2} \equiv \omega
$$

one may determine the celestial expression for $p^{\mu}$, with the result

$$
p^{\mu}=\omega\{1+z \bar{z}, z+\bar{z},-\mathrm{i}(z-\bar{z}), 1-z \bar{z}\},
$$

\footnotetext{
${ }^{2}$ Tools more suitable to handle collinear limits in this context could possibly be devised by considering directly the light-ray operators in the $d=4$ theory. These have a long history in the context of QCD (see, for example, refs. [101, 102]), and have been intensively studied recently in a CFT context (see, for example, refs. [103, 104]). Interestingly, phenomenological applications of these tools to QCD have already started to appear $[105,106]$.
} 
where $z=x z_{1}+(1-x) z_{2}$, and $\omega_{1}=x \omega$, while $\omega_{2}=(1-x) \omega$. Furthermore, $p_{\perp}^{\mu}$ can be written as

$$
\begin{aligned}
p_{\perp}^{\mu}= & \omega x(1-x)\left\{(1-2 x)\left(z_{1} \bar{z}_{2}+\bar{z}_{1} z_{2}\right)+2 x z_{1} \bar{z}_{1}-2(1-x) z_{2} \bar{z}_{2},\right. \\
& \left.z_{12}+\bar{z}_{12},-\mathrm{i}\left(z_{12}-\bar{z}_{12}\right),-(1-2 x)\left(z_{1} \bar{z}_{2}+\bar{z}_{1} z_{2}\right)-2 x z_{1} \bar{z}_{1}+2(1-x) z_{2} \bar{z}_{2}\right\} .
\end{aligned}
$$

The transverse momentum vector $p_{\perp}^{\mu}$ is antisymmetric under the exchange of the labels $1 \leftrightarrow 2$ (which implies $x \leftrightarrow 1-x$ ), as expected from eq. (4.19), and one easily verifies that it satisfies

$$
p_{\perp} \cdot n=p_{\perp} \cdot p=0, \quad p_{\perp}^{2}=-x(1-x) s_{12}=-4 x(1-x) \omega_{1} \omega_{2}\left|z_{12}\right|^{2},
$$

providing a precise connection between the short distance limit on the sphere and the collinear limit in the bulk.

Let us now use the conformal OPE to study the short distance limit of the correlator $\mathcal{C}_{n}$ as, say, $z_{1} \rightarrow z_{2}$. When the locations of the two vertex operators are brought together one finds

$$
: \mathrm{e}^{\mathrm{i} \kappa \mathbf{T}_{1} \cdot \phi\left(z_{1}, \bar{z}_{1}\right)}:: \mathrm{e}^{\mathrm{i} \kappa \mathbf{T}_{2} \cdot \phi\left(z_{2}, \bar{z}_{2}\right)}: \sim\left|z_{12}\right|^{\kappa^{2} \mathbf{T}_{1} \cdot \mathbf{T}_{2}}: \mathrm{e}^{\mathrm{i} \kappa\left(\mathbf{T}_{1}+\mathbf{T}_{2}\right) \cdot \phi(z, \bar{z})}:,
$$

where we placed the field on the r.h.s. of eq. (4.24) at the point $z=x z_{1}+(1-x) z_{2}$ associated with the collinear direction $p^{\mu}$, and corrections are suppressed by powers of $z_{12}$. The $n$-point correlator $\mathcal{C}_{n}$ in this limit is thus re-expressed in terms of the $(n-1)$-point correlator $\mathcal{C}_{n-1}$, where however point $z$ carries the sum of the colour operators of two merging punctures. This is precisely what one expects on the gauge theory side [19, 78], where this property is a consequence of collinear factorisation. The quantity of interest is the splitting anomalous dimension, defined by

$$
\Gamma_{\text {Sp. }}\left(p_{1}, p_{2}\right) \equiv \Gamma_{n}\left(p_{1}, p_{2}, \ldots, p_{n}\right)-\left.\Gamma_{n-1}\left(p, p_{3}, \ldots, p_{n}\right)\right|_{\mathbf{T}_{p} \rightarrow \mathbf{T}_{1}+\mathbf{T}_{2}} .
$$

The short distance limit on the celestial sphere, by means of eq. (4.24), identifies the form of the correlator that one needs to compute in order to analyse the collinear limit in the gauge theory. To make the comparison precise, one needs to extract $\widehat{\Gamma}_{n}^{\text {corr. }}$ from $\mathcal{C}_{n}$, and the appropriate form of $\widehat{\Gamma}_{n-1}^{\text {corr. }}$, with recombined colour factors, from $\mathcal{C}_{n-1}$. Next, one needs to reinstate the dependence on the energies of individual hard particles, since the collinear limit is sensitive to how the energy of the parent particle is subdivided between the two particles forming the collinear pair. At that point, the calculation mirrors exactly the gauge theory procedure: the precise location of the point $z$ on the arc joining $z_{1}$ and $z_{2}$ is irrelevant at leading power in $p_{\perp}$, and the result arises entirely from the scaling of particle energies, $\omega_{1}=x \omega$ and $\omega_{2}=(1-x) \omega$. One finds

$$
\begin{aligned}
\Gamma_{\text {Sp. }}\left(p_{1}, p_{2}\right)=\frac{1}{2} \widehat{\gamma}_{K}\left(\alpha_{s}\right)[ & \ln \left(\frac{-s_{12}+\mathrm{i} \eta}{\mu^{2}}\right) \mathbf{T}_{1} \cdot \mathbf{T}_{2}-\ln x \mathbf{T}_{1} \cdot\left(\mathbf{T}_{1}+\mathbf{T}_{2}\right) \\
& \left.-\ln (1-x) \mathbf{T}_{2} \cdot\left(\mathbf{T}_{1}+\mathbf{T}_{2}\right)\right],
\end{aligned}
$$


in agreement with ref. [19]. One may argue that the result in eq. (4.26) was to some extent implicit in our earlier identification of the soft anomalous dimension matrix with the logarithm of the conformal correlator: we note however that the emerging colour structure is correctly identified by the OPE on the celestial sphere. The result is highly non-trivial in the gauge-theory context: first of all, it embodies the collinear factorisation theorem, stating that the collinear limit depends only on the particles participating in the splitting, and colour correlations with the remaining $n-2$ non-collinear particles vanish at leading power; furthermore, eq. (4.26) is an all-order result, collecting all contributions to $\Gamma_{\mathrm{sp}}$. arising from dipole colour correlations (interesting corrections do of course arise when $\Delta_{n}$ is included).

\section{Some open questions}

It is clear that the construction that we have so far proposed represents only a first step in the exploration of the emerging correspondence, and leaves many open questions. In this section, we begin to discuss some of these questions.

First of all, we note, as we did after eq. (3.6), that the remarkable all-order correspondence between the conformal correlator $\mathcal{C}_{n}$ on the celestial sphere and the infrared operator $\mathcal{Z}_{n}^{\text {corr. }}$ is made possible by the striking factorisation of the coupling and scale dependence from angular and colour variables in eq. (3.7). This raises the question of the nature of the correspondence between the coupling $\kappa$ in the conformal theory and the function $K\left(\alpha_{s}, \epsilon\right)$ in the gauge theory. This question has two aspects, one concerning the choice of the gauge coupling, and the other concerning the integrated scale dependence. With regards to the coupling, we note that one can bypass the need for a perturbative expansion of the function $\widehat{\gamma}_{K}\left(\alpha_{s}\right)$ by simply choosing a scheme in which $\widehat{\gamma}_{K}$ itself plays the role of the coupling. The idea that the universal cusp anomalous dimension should serve as a fundamental gauge coupling, at least in the infrared, is certainly not new, and it has been raised in several contexts (see, for example, refs. [107-111]), providing ample circumstantial evidence that such a choice is physically sensible.

Having taken this option, one must still note that the celestial coupling $\kappa$ does not directly correspond to the new gauge coupling, $\widehat{\gamma}_{K}$, but rather to its 'average' over scales, $K$ in eq. (3.8). We believe that this aspect of the correspondence is dynamically interesting, and deserves closer examination. In a sense, one could argue that any matching between the coupling $\kappa$ on the celestial sphere and the gauge theory must share some of the characteristics of $K$. After all, correlation functions on the sphere are finite and scale-independent, while we are attempting to reproduce infrared divergences in a scaledependent theory: regulator dependence and scale dependence must therefore reside in the matching coefficient. From the gauge theory side, it is quite surprising that such a matching is at all possible, since scale dependence is expected in general to be entangled with colour and kinematics. Even in the case of conformal gauge theories in $d=4$, the analysis of IR divergences requires breaking conformal symmetry by the introduction of an appropriate factorisation scale, much like what happens in the UV case: dimensional regularisation to $d>4$ simply provides an elegant and computationally efficient way to 
perform this symmetry breaking. Indeed, as we noted, all IR divergences of scattering amplitudes, in any gauge theory, are generated by the scale integration, which can be taken to extend to the IR region when $d>4$, while all anomalous dimensions appearing in the scale integrand are finite. That being said, it would be very interesting and appealing if the scale integration could be made part of the correspondence, or, in other words, if one could understand the meaning of the scale integration from the point of view of the conformal theory on the celestial sphere. We note that, from the gauge theory point of view, the IR operator $\mathcal{Z}_{n}$ acts on a hard, finite coefficient function which is located at the common origin of the Wilson lines in eq. (2.14). Distance along the Wilson lines thus can be considered as a proxy for the inverse of the factorisation scale, with infrared scales $(\mu \rightarrow 0)$ located at large distances $(\lambda \rightarrow \infty$ in eq. (2.15)), and ultraviolet scales located at short distances. The scale integration in eq. (3.7), in this picture, could be understood as an integration over a scale factor for the celestial sphere, as it is brought from infinite distance to distance $1 / \mu$. At this stage, of course, these considerations are purely speculative, and they could only be made precise once a more detailed correspondence is constructed.

A second set of considerations must be devoted to the known corrections to eq. (3.7) arising in the gauge theory, i.e. the functions of conformal cross ratios of kinematic invariants that arise starting at the three-loop level, for the scattering of at least four hard particles, and build up the operator $\Delta_{n}$. Clearly, these corrections cannot arise from the free-boson theory that we have just described: the action in eq. (4.1) must be complemented with interaction terms. The nature of these interaction terms is strongly constrained by our knowledge on the gauge theory side of the correspondence. We note, for example, that the three-point correlator in our free-boson theory is trivial, in the sense that it does not contain any colour correlations: indeed, all 'scalar' products $\mathbf{T}_{i} \cdot \mathbf{T}_{j}$ reduce to Casimir eigenvalues, as a consequence of colour conservation, $\mathbf{T}_{1}+\mathbf{T}_{2}+\mathbf{T}_{3}=0$, which implies that $\mathbf{T}_{1} \cdot \mathbf{T}_{2}=\left(C_{3}^{(2)}-C_{1}^{(2)}-C_{2}^{(2)}\right) / 2$, and similarly for the other two cyclic permutations. ${ }^{3}$ When more than three hard particles participate in the scattering, three-point correlations can arise, and one might expect terms of the form $f_{a b c} \mathbf{T}_{1}^{a} \mathbf{T}_{2}^{b} \mathbf{T}_{3}^{c}$ to appear in $\Gamma_{n}$ : indeed, just such correlations do arise at two loops in the scattering of massive hard particles [112117]. No such corrections are possible, however, in the massless theory, essentially because conformal cross-ratios necessarily involve at least four points. The analysis of refs. [31, 33] furthermore shows that three-point correlations do arise in $\Gamma_{n}$ for $n>3$, but they are constrained to be independent of kinematics, and their colour structure has the peculiar form

$$
f_{a b e} f_{c d}^{e}\left\{\mathbf{T}_{i}^{a}, \mathbf{T}_{i}^{d}\right\} \mathbf{T}_{j}^{b} \mathbf{T}_{k}^{c},
$$

with $i \neq j \neq k$; in particular, correlations of this form are necessary to preserve collinear factorisation. The special form of three-point correlations is very relevant to possible generalisations of eq. (4.1), since the bootstrap principle for two-dimensional conformal theories holds that four-point functions of conformal fields are determined once the threepoint functions are given. In the case at hand, of course, the precise form of the full

\footnotetext{
${ }^{3}$ We note in passing that these colour identities guarantee the proper scaling behaviour of three-point functions of vertex operators, consistent with eq. (4.12).
} 
correction to the four-point function at the three-loop level is also known, involving the first 'quadrupole' correction to eq. (3.7). Similar considerations apply to the soft-gluon current in eq. (4.18): in gauge theories, the current has an all-order definition in terms of matrix element of Wilson lines, with a gluon state replacing the vacuum state [118]; this matrix element is known both at one [119] and two loops [120-122], where it exhibits a non-trivial, purely non-abelian colour structure. It is clear that this abundant and detailed knowledge on the gauge-theory side of the correspondence will provide powerful constraints and checks for any attempt to construct the completion of our matrix-valued free-boson proposal.

\section{Perspectives}

The formulation of the infrared problem for gauge theories and gravity in terms of asymptotic symmetries of the $S$-matrix, initiated in refs. [40, 41], provides a novel and interesting viewpoint to investigate long-distance dynamics. One of the most intriguing aspects of this approach is the emergence of a 'holographic' [50] correspondence between certain dynamical properties of massless gauge theories in $d=4$ Minkowski space and their asymptotic behaviour on the celestial sphere at large light-cone times. What emerges is a re-interpretation of the soft limit of gauge amplitudes in terms of a two-dimensional conformal theory defined on the sphere: many elements of this conformal theory can be constructed by taking appropriate limits of gauge-theory quantities, and infrared factorisation properties of loworder perturbative gauge amplitudes can be understood, and in fact generalised, in terms of conformal objects defined on the sphere.

In this paper, inspired by the QED analysis in ref. [69], we took a different viewpoint, starting from the all-order infrared factorisation properties of massless gauge theory amplitudes, and attempting to find a characterisation of the universal infrared operators emerging from the factorisation in terms of a celestial conformal field theory. For simplicity, we have focused on colour correlations of dipole form, which are the only ones appearing up to three loops in the massless theory: we emphasise however that our results apply

to all perturbative orders within this set, and they include both planar and non-planar correlations. A first strong indication that the celestial sphere is an appropriate arena for the discussion of infrared divergences came from the specific form of the exponentiation of kinematic dependence in eq. (3.7): indeed, using the parametrisation in eq. (3.1) exposes a complete factorisation of coupling and scale dependence from kinematic and colour variables, that had not previously been noticed, except in the high-energy limit. The simple and transparent expression in eq. (3.7) provides a strong suggestion for the identification of the appropriate celestial conformal theory, generalising [69] to the non-abelian theory. We find that the all-order gauge-theory result in eq. (3.7) is reproduced by a celestial theory of Lie-algebra-valued free bosons, by computing the correlator of $n$ vertex operators which are gauge-group matrices in the representations of the hard particles participating in the scattering. We have seen that the matrix nature of the vertex operators does not prevent them from being interpreted as conformal fields of definite weight, and we have seen that their correlators have good conformal properties as a consequence of the gauge invariance of the bulk gauge theory. Like $\mathcal{Z}_{n}^{\text {corr. }}$ in eq. (3.7), the conformal correlator must 
be interpreted as a colour operator, defined on the celestial sphere, and acting on the bulk colour degrees of freedom, which can be thought of as being located at the origin. The identification of the celestial conformal field theory is further confirmed by the fact that the conformal OPE reproduces both the tree-level factorisation theorem for the radiation of a soft gluon, and the structure of collinear limits for the hard particles. It is striking that a large fraction of the complexity of non-abelian interactions can be captured by a free field theory on the celestial sphere. Two facts help to clarify this point. First, although the celestial bosons are free, the vertex operators carry charges, which leads to non-trivial interactions: in the abelian limit, the picture is that of a two-dimensional Coulomb gas, with the charges located at the endpoints of the bulk Wilson lines. Second, we note that by restricting our attention to dipole colour correlations we have effectively restricted the interactions of the charges on the sphere to be pairwise. Including multipole correlation will, in all likelihood, require an interacting two-dimensional theory.

In fact, both the known gauge-theory results and the structure of our proposed celestial theory point to the incompleteness of the picture drawn so far. On the celestial side, the fact that we have a local matrix theory suggests that a gauge connection on the sphere might need to be introduced, and consequently the theory is expected to become interacting. While we leave this generalisation to future work, we have noted that known multipole colour correlations arising in the gauge theory starting at the three-loop order will pose stringent constraints on any extension of eq. (4.1). Clearly, the next very significant goal in this program would be to compute the three-loop value of the operator $\Delta_{n}$, first determined in ref. [31], from conformal field theory data. This could open the way to the exploration of even higher-order correlations with novel, and possibly simpler, techniques, not associated with gauge-theory Feynman diagrams. In particular, it would be of great interest to understand, in the conformal context, colour correlations associated with higher-order Casimir operators of the gauge algebra, which have been neglected here, but are known to be present starting at four loops [34, 35, 86-88, 90, 91], and whose role on the gauge-theory side is not yet fully understood.

Aside from this first layer of generalisation, several other interesting questions and possible developments will need to be explored. First of all, the role of collinear singularities should be clarified: the conformal theory, as written, determines only colour correlations, which originate exclusively from soft gluon exchanges at wide angles. Collinear poles are 'colour-singlet' quantities, and are associated to single points on the sphere, and not to arcs. On the other hand, the relatively simple structure of infrared singularities embodied by eq. (2.7) is specific to massless theories and thus it is inextricably linked to the presence of collinear poles.

A related, and certainly more complex question is to what extent this framework could be extended to massive hard particles: in a sense, one would expect drastic complications, since the appropriate asymptotic configuration space would no longer be the punctured Riemann sphere, but rather a three-dimensional hyperbolic space; on the other hand, on the gauge theory side, soft poles are still generated by correlators of semi-infinite Wilson lines, which are scale-invariant even away from the light-cone, and the modifications to the soft anomalous dimension for massive particles at two loops are remarkably 
simple $[112,113,117]$. It is not inconceivable that they might be understood in terms of a deformation of the conformal theory defined in the massless case.

In a final speculative remark, we note that the basic gauge-theoretical ingredients of infrared operators - form factors, matrix elements of Wilson lines and anomalous dimensions - have definitions that apply in principle beyond perturbation theory, and indeed, when strong-coupling data are available, they have been shown to smoothly match onto the perturbative definitions $[123,124]$. If the dictionary mapping these quantities to the celestial sphere can be fully understood, it is possible that non-perturbative features of the four-dimensional theory could be gleaned in the simpler context of the two-dimensional celestial framework. To this end, a more precise matching between the ideas presented here and the well-developed framework initiated in [40, 41] will certainly be useful.

Note added. One week after the present paper was published on arXiv, a paper containing related, partly complementary results appeared [125]. The authors work in the context of celestial amplitudes [55, 56], and show how the infrared factorisation of the momentumspace scattering amplitude is reproduced at the level of Mellin transforms, emphasising the crucial role played by the cusp anomalous dimension for the existence of the celestial amplitude. Their results in the large- $N$ limit, and for the specific cases they consider, are in agreement with our discussion.

\section{Acknowledgments}

This work was partially supported by the Italian Ministry of University and Research (MIUR), grant PRIN 20172LNEEZ. I wholeheartedly thank Claude Duhr for providing the initial motivation for this project, and for important and enlightening discussions. I thank Michele Caselle for sharing his understanding of conformal field theories and for several useful insights. I thank Lance Dixon, Einan Gardi, Marco Meineri, and George Sterman for their reading of the manuscript prior to publication, and for useful and significant observations.

Open Access. This article is distributed under the terms of the Creative Commons Attribution License (CC-BY 4.0), which permits any use, distribution and reproduction in any medium, provided the original author(s) and source are credited.

\section{References}

[1] F. Bloch and A. Nordsieck, Note on the Radiation Field of the electron, Phys. Rev. 52 (1937) 54 [INSPIRE].

[2] T. Kinoshita, Mass singularities of Feynman amplitudes, J. Math. Phys. 3 (1962) 650 [INSPIRE].

[3] T.D. Lee and M. Nauenberg, Degenerate Systems and Mass Singularities, Phys. Rev. 133 (1964) B1549 [INSPIRE].

[4] G.F. Sterman, Mass Divergences in Annihilation Processes. 1. Origin and Nature of Divergences in Cut Vacuum Polarization Diagrams, Phys. Rev. D 17 (1978) 2773 [INSPIRE]. 
[5] G.F. Sterman, Mass Divergences in Annihilation Processes. 2. Cancellation of Divergences in Cut Vacuum Polarization Diagrams, Phys. Rev. D 17 (1978) 2789 [InSPIRE].

[6] P.P. Kulish and L.D. Faddeev, Asymptotic conditions and infrared divergences in quantum electrodynamics, Theor. Math. Phys. 4 (1970) 745 [INSPIRE].

[7] G. Giavarini and G. Marchesini, IR Finite S Matrix in the QCD Coherent State Basis, Nucl. Phys. B 296 (1988) 546 [inSPIRE].

[8] A.H. Mueller, On the Asymptotic Behavior of the Sudakov Form-factor, Phys. Rev. D 20 (1979) 2037 [INSPIRE].

[9] J.C. Collins, Algorithm to Compute Corrections to the Sudakov Form-factor, Phys. Rev. D 22 (1980) 1478 [INSPIRE].

[10] A. Sen, Asymptotic Behavior of the Sudakov Form-Factor in QCD, Phys. Rev. D 24 (1981) 3281 [INSPIRE].

[11] A. Sen, Asymptotic Behavior of the Wide Angle On-Shell Quark Scattering Amplitudes in Nonabelian Gauge Theories, Phys. Rev. D 28 (1983) 860 [inSPIRE].

[12] J.C. Collins, Sudakov form-factors, Adv. Ser. Direct. High Energy Phys. 5 (1989) 573 [hep-ph/0312336] [INSPIRE].

[13] L. Magnea and G.F. Sterman, Analytic continuation of the Sudakov form-factor in QCD, Phys. Rev. D 42 (1990) 4222 [inSPIRE].

[14] S. Catani, The singular behavior of QCD amplitudes at two loop order, Phys. Lett. B 427 (1998) 161 [hep-ph/9802439] [INSPIRE].

[15] G.F. Sterman and M.E. Tejeda-Yeomans, Multiloop amplitudes and resummation, Phys. Lett. B 552 (2003) 48 [hep-ph/0210130] [INSPIRE].

[16] L.J. Dixon, L. Magnea and G.F. Sterman, Universal structure of subleading infrared poles in gauge theory amplitudes, JHEP 08 (2008) 022 [arXiv:0805.3515] [INSPIRE].

[17] T. Becher and M. Neubert, Infrared singularities of scattering amplitudes in perturbative QCD, Phys. Rev. Lett. 102 (2009) 162001 [Erratum ibid. 111 (2013) 199905] [arXiv:0901.0722] [INSPIRE].

[18] E. Gardi and L. Magnea, Factorization constraints for soft anomalous dimensions in $Q C D$ scattering amplitudes, JHEP 03 (2009) 079 [arXiv:0901.1091] [INSPIRE].

[19] T. Becher and M. Neubert, On the Structure of Infrared Singularities of Gauge-Theory Amplitudes, JHEP 06 (2009) 081 [Erratum ibid. 11 (2013) 024] [arXiv:0903.1126] [INSPIRE].

[20] E. Gardi and L. Magnea, Infrared singularities in QCD amplitudes, Nuovo Cim. C 32N5-6 (2009) 137 [arXiv:0908.3273] [INSPIRE].

[21] E. Gardi, J.M. Smillie and C.D. White, The Non-Abelian Exponentiation theorem for multiple Wilson lines, JHEP 06 (2013) 088 [arXiv:1304.7040] [INSPIRE].

[22] I. Feige and M.D. Schwartz, Hard-Soft-Collinear Factorization to All Orders, Phys. Rev. D 90 (2014) 105020 [arXiv:1403.6472] [InSPIRE].

[23] O. Erdoğan and G. Sterman, Ultraviolet divergences and factorization for coordinate-space amplitudes, Phys. Rev. D 91 (2015) 065033 [arXiv:1411.4588] [INSPIRE]. 
[24] Y. Ma, A Forest Formula to Subtract Infrared Singularities in Amplitudes for Wide-angle Scattering, JHEP 05 (2020) 012 [arXiv: 1910.11304] [INSPIRE].

[25] E. Gardi, E. Laenen, G. Stavenga and C.D. White, Webs in multiparton scattering using the replica trick, JHEP 11 (2010) 155 [arXiv:1008.0098] [INSPIRE].

[26] A. Mitov, G. Sterman and I. Sung, Diagrammatic Exponentiation for Products of Wilson Lines, Phys. Rev. D 82 (2010) 096010 [arXiv:1008.0099] [InSPIRE].

[27] E. Gardi and C.D. White, General properties of multiparton webs: Proofs from combinatorics, JHEP 03 (2011) 079 [arXiv: 1102.0756] [INSPIRE].

[28] E. Gardi, J.M. Smillie and C.D. White, On the renormalization of multiparton webs, JHEP 09 (2011) 114 [arXiv:1108.1357] [INSPIRE].

[29] A.A. Vladimirov, Generating function for web diagrams, Phys. Rev. D 90 (2014) 066007 [arXiv:1406.6253] [INSPIRE].

[30] A.A. Vladimirov, Exponentiation for products of Wilson lines within the generating function approach, JHEP 06 (2015) 120 [arXiv: 1501.03316] [INSPIRE].

[31] O. Almelid, C. Duhr and E. Gardi, Three-loop corrections to the soft anomalous dimension in multileg scattering, Phys. Rev. Lett. 117 (2016) 172002 [arXiv:1507.00047] [INSPIRE].

[32] J.M. Henn and B. Mistlberger, Four-Gluon Scattering at Three Loops, Infrared Structure, and the Regge Limit, Phys. Rev. Lett. 117 (2016) 171601 [arXiv:1608.00850] [INSPIRE].

[33] O. Almelid, C. Duhr, E. Gardi, A. McLeod and C.D. White, Bootstrapping the QCD soft anomalous dimension, JHEP 09 (2017) 073 [arXiv: 1706.10162] [INSPIRE].

[34] J.M. Henn, G.P. Korchemsky and B. Mistlberger, The full four-loop cusp anomalous dimension in $\mathcal{N}=4$ super Yang-Mills and QCD, JHEP 04 (2020) 018 [arXiv:1911.10174] [INSPIRE].

[35] A. von Manteuffel, E. Panzer and R.M. Schabinger, Cusp and collinear anomalous dimensions in four-loop QCD from form factors, Phys. Rev. Lett. 124 (2020) 162001 [arXiv: 2002 . 04617] [INSPIRE].

[36] N. Agarwal, A. Danish, L. Magnea, S. Pal and A. Tripathi, Multiparton webs beyond three loops, JHEP 05 (2020) 128 [arXiv: 2003.09714] [INSPIRE].

[37] B. Agarwal, A. von Manteuffel, E. Panzer and R.M. Schabinger, Four-loop collinear anomalous dimensions in $Q C D$ and $\mathcal{N}=4$ super Yang-Mills, arXiv:2102.09725 [INSPIRE].

[38] N. Agarwal, L. Magnea, S. Pal and A. Tripathi, Cwebs beyond three loops in multiparton amplitudes, JHEP 03 (2021) 188 [arXiv:2102.03598] [INSPIRE].

[39] W.J. Torres Bobadilla et al., May the four be with you: Novel IR-subtraction methods to tackle NNLO calculations, Eur. Phys. J. C 81 (2021) 250 [arXiv:2012.02567] [INSPIRE].

[40] A. Strominger, Asymptotic Symmetries of Yang-Mills Theory, JHEP 07 (2014) 151 [arXiv: 1308.0589] [INSPIRE].

[41] A. Strominger, On BMS Invariance of Gravitational Scattering, JHEP 07 (2014) 152 [arXiv: 1312.2229] [INSPIRE].

[42] H. Bondi, M.G.J. van der Burg and A.W.K. Metzner, Gravitational waves in general relativity. 7. Waves from axisymmetric isolated systems, Proc. Roy. Soc. Lond. A 269 (1962) 21. 
[43] R.K. Sachs, Gravitational waves in general relativity. 8. Waves in asymptotically flat space-times, Proc. Roy. Soc. Lond. A 270 (1962) 103.

[44] V. Lysov, S. Pasterski and A. Strominger, Low's Subleading Soft Theorem as a Symmetry of QED, Phys. Rev. Lett. 113 (2014) 111601 [arXiv:1407.3814] [InSPIRE].

[45] T. He, P. Mitra, A.P. Porfyriadis and A. Strominger, New Symmetries of Massless QED, JHEP 10 (2014) 112 [arXiv:1407.3789] [InSPIRE].

[46] T. He, P. Mitra and A. Strominger, 2D Kac-Moody Symmetry of 4 D Yang-Mills Theory, JHEP 10 (2016) 137 [arXiv:1503.02663] [InSPIRE].

[47] T. Adamo and E. Casali, Perturbative gauge theory at null infinity, Phys. Rev. D 91 (2015) 125022 [arXiv: 1504.02304] [INSPIRE].

[48] A. Strominger, Magnetic Corrections to the Soft Photon Theorem, Phys. Rev. Lett. 116 (2016) 031602 [arXiv: 1509.00543] [inSPIRE].

[49] B. Gabai and A. Sever, Large gauge symmetries and asymptotic states in QED, JHEP 12 (2016) 095 [arXiv: 1607.08599] [INSPIRE].

[50] C. Cheung, A. de la Fuente and R. Sundrum, $4 D$ scattering amplitudes and asymptotic symmetries from 2D CFT, JHEP 01 (2017) 112 [arXiv: 1609.00732] [INSPIRE].

[51] A. Nande, M. Pate and A. Strominger, Soft Factorization in QED from $2 D$ Kac-Moody Symmetry, JHEP 02 (2018) 079 [arXiv:1705.00608] [INSPIRE].

[52] A. Strominger, Lectures on the Infrared Structure of Gravity and Gauge Theory, arXiv: 1703.05448 [INSPIRE].

[53] S. Albayrak, C. Chowdhury and S. Kharel, On loop celestial amplitudes for gauge theory and gravity, Phys. Rev. D 102 (2020) 126020 [arXiv:2007.09338] [INSPIRE].

[54] H.A. González, A. Puhm and F. Rojas, Loop corrections to celestial amplitudes, Phys. Rev. D 102 (2020) 126027 [arXiv: 2009.07290] [INSPIRE].

[55] S. Pasterski, S.-H. Shao and A. Strominger, Flat Space Amplitudes and Conformal Symmetry of the Celestial Sphere, Phys. Rev. D 96 (2017) 065026 [arXiv:1701.00049] [INSPIRE].

[56] S. Pasterski and S.-H. Shao, Conformal basis for flat space amplitudes, Phys. Rev. D 96 (2017) 065022 [arXiv:1705.01027] [INSPIRE].

[57] S. Pasterski, S.-H. Shao and A. Strominger, Gluon Amplitudes as 2d Conformal Correlators, Phys. Rev. D 96 (2017) 085006 [arXiv:1706.03917] [InSPIRE].

[58] A. Schreiber, A. Volovich and M. Zlotnikov, Tree-level gluon amplitudes on the celestial sphere, Phys. Lett. B 781 (2018) 349 [arXiv:1711.08435] [INSPIRE].

[59] S. Stieberger and T.R. Taylor, Symmetries of Celestial Amplitudes, Phys. Lett. B 793 (2019) 141 [arXiv: 1812.01080] [INSPIRE].

[60] E. Himwich and A. Strominger, Celestial current algebra from Low's subleading soft theorem, Phys. Rev. D 100 (2019) 065001 [arXiv:1901.01622] [INSPIRE].

[61] W. Fan, A. Fotopoulos and T.R. Taylor, Soft Limits of Yang-Mills Amplitudes and Conformal Correlators, JHEP 05 (2019) 121 [arXiv: 1903.01676] [INSPIRE].

[62] M. Pate, A.-M. Raclariu and A. Strominger, Conformally Soft Theorem in Gauge Theory, Phys. Rev. D 100 (2019) 085017 [arXiv:1904.10831] [INSPIRE]. 
[63] D. Nandan, A. Schreiber, A. Volovich and M. Zlotnikov, Celestial Amplitudes: Conformal Partial Waves and Soft Limits, JHEP 10 (2019) 018 [arXiv:1904.10940] [InSPIRE].

[64] A. Fotopoulos and T.R. Taylor, Primary Fields in Celestial CFT, JHEP 10 (2019) 167 [arXiv: 1906.10149] [INSPIRE].

[65] S. Ebert, A. Sharma and D. Wang, Descendants in celestial CFT and emergent multi-collinear factorization, JHEP 03 (2021) 030 [arXiv: 2009.07881] [INSPIRE].

[66] N. Arkani-Hamed, M. Pate, A.-M. Raclariu and A. Strominger, Celestial Amplitudes from $U V$ to $I R$, arXiv: 2012.04208 [INSPIRE].

[67] W. Fan, A. Fotopoulos, S. Stieberger, T.R. Taylor and B. Zhu, Conformal Blocks from Celestial Gluon Amplitudes, arXiv:2103.04420 [INSPIRE].

[68] D. Neill and F. Ringer, Soft Fragmentation on the Celestial Sphere, JHEP 06 (2020) 086 [arXiv: 2003.02275] [INSPIRE].

[69] N. Kalyanapuram, Soft Gravity by Squaring Soft QED on the Celestial Sphere, Phys. Rev. D 103 (2021) 085016 [arXiv: 2011.11412] [INSPIRE].

[70] S. Weinberg, Infrared photons and gravitons, Phys. Rev. 140 (1965) B516 [InSPIRE].

[71] G.P. Korchemsky, On near forward high-energy scattering in QCD, Phys. Lett. B $\mathbf{3 2 5}$ (1994) 459 [hep-ph/9311294] [INSPIRE].

[72] I.A. Korchemskaya and G.P. Korchemsky, High-energy scattering in QCD and cross singularities of Wilson loops, Nucl. Phys. B 437 (1995) 127 [hep-ph/9409446] [INSPIRE].

[73] I.A. Korchemskaya and G.P. Korchemsky, Evolution equation for gluon Regge trajectory, Phys. Lett. B 387 (1996) 346 [hep-ph/9607229] [INSPIRE].

[74] V. Del Duca, C. Duhr, E. Gardi, L. Magnea and C.D. White, An infrared approach to Reggeization, Phys. Rev. D 85 (2012) 071104 [arXiv:1108.5947] [InSPIRE].

[75] V. Del Duca, C. Duhr, E. Gardi, L. Magnea and C.D. White, The infrared structure of gauge theory amplitudes in the high-energy limit, JHEP 12 (2011) 021 [arXiv:1109.3581] [INSPIRE].

[76] Z. Bern, J.J.M. Carrasco and H. Johansson, New Relations for Gauge-Theory Amplitudes, Phys. Rev. D 78 (2008) 085011 [arXiv: 0805.3993] [InSPIRE].

[77] Z. Bern, J.J. Carrasco, M. Chiodaroli, H. Johansson and R. Roiban, The Duality Between Color and Kinematics and its Applications, arXiv:1909.01358 [INSPIRE].

[78] S. Catani, D. de Florian and G. Rodrigo, The triple collinear limit of one loop $Q C D$ amplitudes, Phys. Lett. B 586 (2004) 323 [hep-ph/0312067] [INSPIRE].

[79] A. Bassetto, M. Ciafaloni and G. Marchesini, Jet Structure and Infrared Sensitive Quantities in Perturbative QCD, Phys. Rept. 100 (1983) 201 [INSPIRE].

[80] S. Catani and M.H. Seymour, A general algorithm for calculating jet cross-sections in NLO QCD, Nucl. Phys. B 485 (1997) 291 [Erratum ibid. 510 (1998) 503] [hep-ph/9605323] [INSPIRE].

[81] V.S. Dotsenko and S.N. Vergeles, Renormalizability of Phase Factors in the Nonabelian Gauge Theory, Nucl. Phys. B 169 (1980) 527 [InSPIRE].

[82] R.A. Brandt, F. Neri and M.-a. Sato, Renormalization of Loop Functions for All Loops, Phys. Rev. D 24 (1981) 879 [INSPIRE]. 
[83] G.P. Korchemsky and A.V. Radyushkin, Loop Space Formalism and Renormalization Group for the Infrared Asymptotics of QCD, Phys. Lett. B 171 (1986) 459 [inSPIRE].

[84] G.P. Korchemsky and A.V. Radyushkin, Infrared asymptotics of perturbative QCD: Renormalization properties of the wilson loops in higher orders of perturbation theory, Sov. J. Nucl. Phys. 44 (1986) 877 [inSPIRE].

[85] G.P. Korchemsky and A.V. Radyushkin, Renormalization of the Wilson Loops Beyond the Leading Order, Nucl. Phys. B 283 (1987) 342 [INSPIRE].

[86] S. Moch, B. Ruijl, T. Ueda, J.A.M. Vermaseren and A. Vogt, On quartic colour factors in splitting functions and the gluon cusp anomalous dimension, Phys. Lett. B 782 (2018) 627 [arXiv: 1805.09638] [INSPIRE].

[87] A. Grozin, J. Henn and M. Stahlhofen, On the Casimir scaling violation in the cusp anomalous dimension at small angle, JHEP 10 (2017) 052 [arXiv: 1708.01221] [INSPIRE].

[88] J.M. Henn, T. Peraro, M. Stahlhofen and P. Wasser, Matter dependence of the four-loop cusp anomalous dimension, Phys. Rev. Lett. 122 (2019) 201602 [arXiv:1901.03693] [INSPIRE].

[89] G. Falcioni, E. Gardi, C. Milloy and L. Vernazza, Climbing three-Reggeon ladders: four-loop amplitudes in the high-energy limit in full colour, arXiv:2012.00613 [INSPIRE].

[90] V. Ahrens, M. Neubert and L. Vernazza, Structure of Infrared Singularities of Gauge-Theory Amplitudes at Three and Four Loops, JHEP 09 (2012) 138 [arXiv: 1208.4847] [INSPIRE].

[91] T. Becher and M. Neubert, Infrared singularities of scattering amplitudes and $N^{3} L L$ resummation for $n$-jet processes, JHEP 01 (2020) 025 [arXiv: 1908.11379] [INSPIRE].

[92] F.C.S. Brown, Polylogarithmes multiples uniformes en une variable, Compt. Rend. Math. 338 (2004) 527 [INSPIRE].

[93] O. Schnetz, Graphical functions and single-valued multiple polylogarithms, Commun. Num. Theor. Phys. 08 (2014) 589 [arXiv:1302.6445] [INSPIRE].

[94] Z. Bern, L.J. Dixon and V.A. Smirnov, Iteration of planar amplitudes in maximally supersymmetric Yang-Mills theory at three loops and beyond, Phys. Rev. D 72 (2005) 085001 [hep-th/0505205] [INSPIRE].

[95] L. Magnea, Analytic resummation for the quark form-factor in QCD, Nucl. Phys. B 593 (2001) 269 [hep-ph/0006255] [INSPIRE].

[96] V. Del Duca, G. Falcioni, L. Magnea and L. Vernazza, Analyzing high-energy factorization beyond next-to-leading logarithmic accuracy, JHEP 02 (2015) 029 [arXiv:1409.8330] [INSPIRE].

[97] P.H. Ginsparg, Applied Conformal Field Theory, in Les Houches Summer School in Theoretical Physics: Fields, Strings, Critical Phenomena, (1988) [hep-th/9108028] [INSPIRE].

[98] P. Goddard and D.I. Olive, Kac-Moody and Virasoro Algebras in Relation to Quantum Physics, Int. J. Mod. Phys. A 1 (1986) 303 [inSPIRE].

[99] S. Caron-Huot, When does the gluon reggeize?, JHEP 05 (2015) 093 [arXiv:1309.6521] [INSPIRE]. 
[100] J. Polchinski, String theory. Vol. 1: An introduction to the bosonic string, Cambridge Monographs on Mathematical Physics, Cambridge University Press (2007), [DOI] [INSPIRE].

[101] I.I. Balitsky and V.M. Braun, Evolution Equations for QCD String Operators, Nucl. Phys. B 311 (1989) 541 [INSPIRE].

[102] V.M. Braun, G.P. Korchemsky and D. Müller, The uses of conformal symmetry in QCD, Prog. Part. Nucl. Phys. 51 (2003) 311 [hep-ph/0306057] [inSPIRE].

[103] P. Kravchuk and D. Simmons-Duffin, Light-ray operators in conformal field theory, JHEP 11 (2018) 102 [arXiv:1805.00098] [INSPIRE].

[104] M. Kologlu, P. Kravchuk, D. Simmons-Duffin and A. Zhiboedov, The light-ray OPE and conformal colliders, JHEP 01 (2021) 128 [arXiv: 1905.01311] [INSPIRE].

[105] H. Chen, I. Moult and H.X. Zhu, Quantum Interference in Jet Substructure from Spinning Gluons, Phys. Rev. Lett. 126 (2021) 112003 [arXiv:2011.02492] [INSPIRE].

[106] H. Chen, I. Moult and H.X. Zhu, Spinning Gluons from the QCD Light-Ray OPE, arXiv:2104.00009 [INSPIRE].

[107] S. Catani, B.R. Webber and G. Marchesini, $Q C D$ coherent branching and semiinclusive processes at large $x$, Nucl. Phys. B 349 (1991) 635 [inSPIRE].

[108] O. Erdoğan and G. Sterman, Gauge Theory Webs and Surfaces, Phys. Rev. D 91 (2015) 016003 [arXiv: 1112.4564] [INSPIRE].

[109] A. Grozin, J.M. Henn, G.P. Korchemsky and P. Marquard, The three-loop cusp anomalous dimension in QCD and its supersymmetric extensions, JHEP 01 (2016) 140 [arXiv: 1510.07803] [INSPIRE].

[110] A. Banfi, B.K. El-Menoufi and P.F. Monni, The Sudakov radiator for jet observables and the soft physical coupling, JHEP 01 (2019) 083 [arXiv: 1807.11487] [INSPIRE].

[111] S. Catani, D. De Florian and M. Grazzini, Soft-gluon effective coupling and cusp anomalous dimension, Eur. Phys. J. C 79 (2019) 685 [arXiv:1904.10365] [INSPIRE].

[112] T. Becher and M. Neubert, Infrared singularities of QCD amplitudes with massive partons, Phys. Rev. D 79 (2009) 125004 [Erratum ibid. 80 (2009) 109901] [arXiv:0904.1021] [INSPIRE].

[113] A. Ferroglia, M. Neubert, B.D. Pecjak and L.L. Yang, Two-loop divergences of scattering amplitudes with massive partons, Phys. Rev. Lett. 103 (2009) 201601 [arXiv:0907.4791] [INSPIRE].

[114] A. Ferroglia, M. Neubert, B.D. Pecjak and L.L. Yang, Two-loop divergences of massive scattering amplitudes in non-abelian gauge theories, JHEP 11 (2009) 062 [arXiv:0908.3676] [INSPIRE].

[115] A. Mitov, G.F. Sterman and I. Sung, The Massive Soft Anomalous Dimension Matrix at Two Loops, Phys. Rev. D 79 (2009) 094015 [arXiv:0903.3241] [INSPIRE].

[116] N. Kidonakis, Two-loop soft anomalous dimensions and NNLL resummation for heavy quark production, Phys. Rev. Lett. 102 (2009) 232003 [arXiv:0903.2561] [INSPIRE].

[117] Y.-T. Chien, M.D. Schwartz, D. Simmons-Duffin and I.W. Stewart, Jet Physics from Static Charges in AdS, Phys. Rev. D 85 (2012) 045010 [arXiv:1109.6010] [INSPIRE]. 
[118] L. Magnea, E. Maina, G. Pelliccioli, C. Signorile-Signorile, P. Torrielli and S. Uccirati, Factorisation and Subtraction beyond NLO, JHEP 12 (2018) 062 [arXiv:1809.05444] [INSPIRE].

[119] S. Catani and M. Grazzini, The soft gluon current at one loop order, Nucl. Phys. B 591 (2000) 435 [hep-ph/0007142] [INSPIRE].

[120] S.D. Badger and E.W.N. Glover, Two loop splitting functions in QCD, JHEP 07 (2004) 040 [hep-ph/0405236] [INSPIRE].

[121] C. Duhr and T. Gehrmann, The two-loop soft current in dimensional regularization, Phys. Lett. B 727 (2013) 452 [arXiv: 1309.4393] [InSPIRE].

[122] Y. Li and H.X. Zhu, Single soft gluon emission at two loops, JHEP 11 (2013) 080 [arXiv: 1309.4391] [INSPIRE].

[123] L.F. Alday and J.M. Maldacena, Gluon scattering amplitudes at strong coupling, JHEP 06 (2007) 064 [arXiv: 0705.0303] [INSPIRE].

[124] L.F. Alday, Universal structure of subleading infrared poles at strong coupling, JHEP 07 (2009) 047 [arXiv: 0904.3983] [InSPIRE].

[125] H.A. González and F. Rojas, The structure of IR divergences in celestial gluon amplitudes, arXiv:2104.12979 [INSPIRE]. 